\title{
Baroclinic Structure of Oceanic Rings
}

\author{
L. Zhang ${ }^{1,2}$, C. Sun ${ }^{1 *}$ \\ ${ }^{1}$ Institute of Occanology, Chinese Academy of Sciences, Qingdao 266071, China \\ ${ }^{2}$ Graduate School of Chinese Academy of Sciences, Beijing 100039, China \\ Email: csun âms.qdio.ac.cn
}

\begin{abstract}
One of the most important analytical solutions to the two dimensional incompressible flow is circular Rankin vortex that has a solid-body core and an approximately irrotational far field. For an f-plane rotating flow, Ingersoll (1969) presented a closed-form ring solution superposed with a zonal flow. So far most studies have been concerned with two dimensional and barotropic situations.

One oceanic phenomenon resembling circular vortex is ring, which is a meso-scale flow structure associated with an isolated mass of anomalous water, significantly warmer or colder, saltier or fresher than its surroundings. During the past decades, much attention has been devoted to the observation of rings in the ocean. Joyce [1] presented the vertical structure of velocity and other hydrographic properties of a Gulf Stream warm core ring, and found that in the horizontal layer the central core was rotating as a solid body approximately once every 3.6 days, and the azimuthal velocity of the outside decreased with radius, in the vertical direction the azimuthal velocity decreased with depth below $100 \mathrm{~m}$. These studies reveal the baroclinic structure of oceanic rings that can not be described by conventional $2 \mathrm{D}$ vortex solution.

To account for the stratified oceanic field, some simple layer-models are constructed to describe the observed velocity distribution of rings, among which Rubino's [2] five-layer, reduced-gravity mode] is a recent example, which presents a vertically stratified ring characterized by azimuthal velocity fields that are nonlinear functions of the radius. Although layer-model is a progress compared with barotropic solution, it is inadequate for describing baroclinic features in continuously stratified fluid. In this study, we are motivated by recent observations of geostrophic empirical mode in oceanic and atmospheric jets [3] and develop an f-plane baroclinic vortex solution that is esscntially equivalentbarotropic and capable of describing the observed three-dimensional velocity and density distributions in oceanic rings. Unlike the previous layer-model approach, the GEM vortex solution is intended for continuously stratified fluids. It allows us to conduct a series of numerical experiments with general circulation model. The phenomenon that geophysical vortex tends to evolve to a vertical alignment state with horizontally axisymmetric shape is also investigated.
\end{abstract}

\section{REFERENCES}

1. Joyce TM. Velocity and hydrographic structure of a Gulf Stream warm-core ring. J. Phys. Oceanogr., 1984;14:936-947

2. Dotsenko S, Rubino A. Analytical solutions for circular stratified eddies of the reduced-gravity shallow-water equations. J. Phys. Oceanogr., 2006;36:1693-1702

3. Sun C, Watts D. A circumpolar gravest empirical mode for the Southern Ocean hydrography. $J$. Geophys. Res., 2001;106:2833-2855 